\title{
Two Integrable Cases of a Ball Rolling over a Sphere in $\mathbb{R}^{n}$
}

\author{
B. Gajić, B. Jovanović
}

We consider the nonholonomic problem of rolling without slipping and twisting of a balanced ball over a fixed sphere in $\mathbb{R}^{n}$. By relating the system to a modified LR system, we prove that the problem always has an invariant measure. Moreover, this is a $S O(n)$-Chaplygin system that reduces to the cotangent bundle $T^{*} S^{n-1}$. We present two integrable cases. The first one is obtained for a special inertia operator that allows the Chaplygin Hamiltonization of the reduced system. In the second case, we consider the rigid body inertia operator $\mathbb{I} \omega=I \omega+\omega I$, $I=\operatorname{diag}\left(I_{1}, \ldots, I_{n}\right)$ with a symmetry $I_{1}=I_{2}=\ldots=I_{r} \neq I_{r+1}=I_{r+2}=\ldots=I_{n}$. It is shown that general trajectories are quasi-periodic, while for $r \neq 1, n-1$ the Chaplygin reducing multiplier method does not apply.

Keywords: nonholonomic Chaplygin systems, invariant measure, integrability

\section{Introduction}

1. Chaplygin systems. Let $(Q, L, \mathcal{D})$ be a nonholonomic system, where $Q$ is a configuration space and $\mathcal{D}$ a nonintegrable distribution of constraints. Assume that the Lie group $G$ acts freely $Q, L$ is $G$-invariant, the quotient space $N=Q / G$ is a manifold, and $\mathcal{D}$ is $G$-invariant, transversal and a complement to the $G$-orbits ( $\mathcal{D}$ is a principal connection of the bundle $Q \rightarrow N=M / G)$. Then the nonholonomic system is $G$-invariant and reduces to the tangent bundle of the base manifold $N$. The reduced Lagrange-d'Alembert equations take the form

$$
\left(\frac{\partial L_{r e d}}{\partial x}-\frac{d}{d t} \frac{\partial L_{r e d}}{\partial \dot{x}}, \eta\right)=\Sigma(\dot{x}, \dot{x}, \eta) \quad \text { for all } \quad \eta \in T_{x} N
$$

Received June 26, 2019

Accepted August 28, 2019

This research was supported by the Serbian Ministry of Science Project 174020, Geometry and Topology of Manifolds, Classical Mechanics and Integrable Dynamical Systems.

Borislav Gajić

gajab@mi.sanu.ac.rs

Božidar Jovanović

bozaj@mi.sanu.ac.rs

Mathematical Institute SANU,

Kneza Mihaila 36, 11000, Belgrade, Serbia 
where the reduced Lagrangian $L_{\text {red }}$ is obtained from $\left.L\right|_{\mathcal{D}}$ by the identification $T N=\mathcal{D} / G$, and $\Sigma(X, Y, Z)$ is a $(0,3)$-tensor field on the base manifold $N$, skew-symmetric in $Y$ and $Z$, which depends on the metric and the curvature of the connection. The system $(Q, L, \mathcal{D}, G)$ is referred to as a G-Chaplygin system, as a generalization of classical Chaplygin systems with Abelian symmetries $[2,12,14,34]$.

For $\Sigma \neq 0$ the equations are not Hamiltonian. Chaplygin proposed a remarkable Hamiltonization procedure as follows [14]. Consider the time substitution $d \tau=\nu(x) d t$, and denote $x^{\prime}=d x / d \tau=\dot{x} / \nu$. Then the Lagrangian function transforms to $L^{*}\left(x^{\prime}, x\right)=\left.L_{\text {red }}(\dot{x}, x)\right|_{\dot{x}=\nu x^{\prime}}$. The factor $\nu$ is a Chaplygin reducing multiplier if in the new time $\tau$ the reduced system (1.1) transforms into the usual Euler-Lagrange equations of $L^{*}\left(x^{\prime}, x\right)$. In particular, in the cotangent bundle formulation, the original system has an invariant measure and it is conformally symplectic (see $[3,12,20]$ ).

The aim of this survey is to provide an example of a system with an invariant measure, such that the reduced system in the first class of metrics can be solved by using the Chaplygin reducing multiplier method, while for the second class it does not allow a Chaplygin multiplier, but is still solvable. Our exposition follows the results published in [24, 27, 30, 33].

2. Motivation. One of the basic classical nonholonomic problems is rolling without slipping of a nonhomogeneous balanced ball over the fixed horizontal plane. Chaplygin derived the equations and reduced the problem to the Poisson sphere. He also proved that the system is integrable using sphero-conical coordinates (see [13]). After [13], a balanced ball (with the inertia operator that is not proportional to the identity operator) is usually referred as a Chaplygin ball.

There are several possible generalizations of this system. For example, the higher-dimensional variant of the problem is considered in [21, 28]. On the other hand, the problem of rolling of the Chaplygin ball of radius $\rho$ over a fixed sphere of radius $\sigma$ is considered in [7,8]. It is shown that the system possesses an invariant measure. However, for different moments of inertia $\left(I_{1} \neq I_{2} \neq I_{3}\right)$, its integrability is proved only when parameter

$$
\epsilon=\frac{\sigma}{\sigma \pm \rho}
$$

equals -1 . This remarkable case represents the rolling of a spherical shell over a fixed sphere placed inside, such that the radius of the shell is twice the radius of the sphere.

The problem of rolling of the Chaplygin ball over a fixed sphere with the additional condition that the angular velocity of the ball belongs to the common tangent plane of the ball and the sphere (rubber rolling) is introduced by Ehlers and Koiller in [17].

Let $\mathbb{I}=\operatorname{diag}\left(I_{1}, I_{2}, I_{3}\right), \vec{\omega}, m$ be the inertia operator, angular velocity, and mass of the ball, and let $\vec{\gamma}$ be the unit normal on the sphere at the point of contact. In the vector notation, the equation of the system can be written in the form

$$
\dot{\vec{m}}=\vec{m} \times \vec{\omega}+\lambda \vec{\gamma}, \quad \dot{\vec{\gamma}}=\epsilon \vec{\gamma} \times \vec{\omega},
$$

where

$$
\vec{m}=\left(\mathbb{I}+m \rho^{2} \mathbb{E}\right) \vec{\omega}=\mathbf{I} \vec{\omega}, \quad \lambda=-\left(\vec{m}, \mathbf{I}^{-1}(\vec{m} \times \vec{\omega})\right) /\left(\vec{\gamma}, \mathbf{I}^{-1} \vec{\gamma}\right) .
$$

The density of an invariant measure in coordinates $(\omega, \gamma)$ reads

$$
\mu_{\epsilon}(\vec{\gamma})=\left(\mathbf{I}^{-1} \vec{\gamma}, \vec{\gamma}\right)^{\frac{1}{2 \epsilon}}
$$

(see [17]). As in the case of nonrubber rolling, when the moments of inertia are different, Borisov and Mamaev proved the integrability for $\epsilon=-1$ [9]. In order to reduce both problems (with 
and without rubber condition) to quadratures, in $[8,9]$ the special nonholonomic deformations of the sphero-conical coordinates are constructed. Note that in the limiting case, when $\epsilon=1$, the above equations coincide with the equations of nonholonomic rigid body motion studied by Veselov and Veselova [39, 40]. Also, in a symmetric case $I_{1}=I_{2}$, the system is integrable for all $\epsilon[4]$.

3. Outline. The system (1.3) suggests that it is natural to consider Euler-Poincaré equations with constraints on Lie algebras with modified Poisson type equations. This is performed in [30] where it is proved that the corresponding flows possess invariant measures (Section 2). Further, the papers of Borisov, Mamaev and Ehlers, Koiller were our motivation for the study of the rubber rolling of a ball over the fixed sphere in $\mathbb{R}^{n}$ (see [33]). It is shown that the system is a $S O(n)$-Chaplygin system with an invariant measure (Section 3 ).

For a special choice of inertia operator, combining the Chaplygin reducing multiplier and the Maupertuis principle, in [24] the reduced system is transformed to the zero-energy level set of a natural mechanical system on a sphere endowed with the standard metric and the potential $V_{\epsilon}(x)=-\left(A^{-1} x, x\right)^{-1 / \epsilon}$, with $A=\operatorname{diag}\left(a_{1}, \ldots, a_{n}\right)$. In particular, we have integrability in the limiting case $\epsilon=+1$ when one gets Braden's potential [11], and for $\epsilon=-1$ when one gets a celebrated Neumann's potential [35] (Section 4). In [27] the same system is considered with the rigid body inertia operator $\mathbb{I} \omega=I \omega+\omega I, I=\operatorname{diag}\left(I_{1}, \ldots, I_{n}\right)$. In a symmetric case $I_{1}=I_{2}=\ldots=I_{r} \neq I_{r+1}=I_{r+2}=\ldots=I_{n}$, the reduced system is quasi-periodic over two- and three-dimensional tori. It is interesting that additional integrals, even if they are similar to the Noether integrals, are not obtained from the nonholonomic version of the Noether theorem. Moreover, the system does not admit the Chaplygin multiplier for $r \neq 1, n-1$ and $\epsilon \neq 1 / 2$ (Section 5).

\section{Modified LR systems}

1. Definition and an invariant measure. Let $\mathfrak{g}$ be a $n$-dimensional reductive Lie algebra, with an invariant scalar product $\langle\cdot, \cdot\rangle$, which we use to identify $\mathfrak{g}$ and $\mathfrak{g}^{*}$. Let $\mathbb{I}: \mathfrak{g} \rightarrow \mathfrak{g}$ be a positive definite operator. As the ball-sphere problem (1.3) suggests, it is natural to consider $\epsilon L R$ system on the space $\mathfrak{g}^{k+1}\left(\omega, e_{1}, \ldots, e_{k}\right)$ defined by the equations

$$
\begin{aligned}
& \dot{m}=[m, \omega]+\Lambda, \quad \Lambda=\sum_{i=1}^{k} \lambda^{i} e_{i}, \quad m=\mathbb{I} \omega, \\
& \dot{e}_{i}=\epsilon\left[e_{i}, \omega\right],
\end{aligned}
$$

$i=1, \ldots, k$. The term $\Lambda$ can be interpreted as a reaction force and it is determined from the condition that the trajectories $\left(\omega(t), e_{1}(t), \ldots, e_{k}(t)\right)$ satisfy constraints

$$
\phi_{i}=\left\langle\omega, e_{i}\right\rangle=c_{i}=\text { const }, \quad i=1, \ldots, k .
$$

By differentiating (2.3), we obtain the linear system

$$
\epsilon\left\langle\left[e_{i}, \omega\right], \omega\right\rangle+\left\langle e_{i}, \mathbb{I}^{-1}[m, \omega]\right\rangle+\sum_{j} \lambda^{j} \mathbb{A}_{i j}=0, \quad \mathbb{A}_{i j}=\left\langle e_{i}, \mathbb{I}^{-1} e_{j}\right\rangle,
$$

and we get the Lagrange multipliers in the form

$$
\lambda^{i}=-\sum_{j}\left\langle e_{j}, \mathbb{I}^{-1}[m, \omega]\right\rangle \mathbb{A}^{i j}
$$


Here $\mathbb{A}^{i j}$ is the inverse of the matrix $\mathbb{A}_{i j}=\left\langle\mathbb{I}^{-1} e_{i}, e_{j}\right\rangle, i, j=1, \ldots, k$. In particular, $\Lambda$ does not depend on $\epsilon$.

Theorem 1 ([30]). The $\epsilon$ LR system (2.1), (2.2), (2.5) has an invariant measure

$$
\mu_{\epsilon} \mathrm{d} \omega \wedge \mathrm{d} e_{1} \wedge \ldots \wedge \mathrm{d} e_{k}
$$

where the density is given by

$$
\mu_{\epsilon}=(\Delta)^{\frac{1}{2 \epsilon}}, \quad \Delta=\operatorname{det}\left(\mathbb{A}_{i j}\right)=\operatorname{det}\left(\left\langle\mathbb{I}^{-1} e_{i}, e_{j}\right\rangle\right), \quad i, j=1, \ldots, k .
$$

For $\epsilon=1$, the system is known as the LR system (see [39, 40]) and the density (2.7) reduces to the one given in [40].

2. Momentum equation. It is clear that we can also consider the $\epsilon \operatorname{LR}$ system (2.1), $(2.2),(2.5)$ on the space

$$
\mathcal{M}=\left\{\left(\omega, e_{1}, \ldots, e_{n}\right) \in \mathfrak{g}^{n+1} \mid\left\langle e_{i}, e_{j}\right\rangle=\delta_{i j}\right\},
$$

simply by taking $i=1, \ldots, n$ in Eq. (2.2). Then the system has an invariant measure

$$
\left.\mu_{\epsilon} \mathrm{d} \omega \wedge \mathrm{d} e_{1} \wedge \ldots \wedge \mathrm{d} e_{n}\right|_{\mathcal{M}}
$$

Moreover, on $\mathcal{M}$ we have well-defined orthogonal projections $\operatorname{pr}_{\mathcal{H}}, \operatorname{pr}_{\mathcal{D}}\left(\operatorname{pr}_{\mathcal{H}}+\operatorname{pr}_{\mathcal{D}}=\mathbb{E}\right)$ onto the complementary subspaces

$$
\mathcal{H}=\operatorname{span}\left\{\mathrm{e}_{1}, \ldots, \mathrm{e}_{\mathrm{k}}\right\}, \quad \mathcal{D}=\operatorname{span}\left\{\mathrm{e}_{\mathrm{k}+1}, \ldots, \mathrm{e}_{\mathrm{n}}\right\},
$$

which, according to (2.2), satisfy the equations

$$
\frac{d}{d t} \operatorname{pr}_{\mathcal{D}}=\epsilon\left[\operatorname{pr}_{\mathcal{D}}, \operatorname{ad}_{\omega}\right], \quad \frac{d}{d t} \operatorname{pr}_{\mathcal{H}}=\epsilon\left[\operatorname{pr}_{\mathcal{H}}, \operatorname{ad}_{\omega}\right] .
$$

Note that, with the above notation, we can express $\Lambda$ in (2.1) as

$$
\Lambda=-\mathbb{A}^{-1} \operatorname{pr}_{\mathcal{H}} \mathbb{I}^{-1}[\mathbb{I} \omega, \omega],
$$

where $\mathbb{A}^{-1}: \mathcal{H} \rightarrow \mathcal{H}$ is the inverse of the mapping $\mathbb{A}=\left.\mathbb{I}^{-1}\right|_{\mathcal{H}}=\operatorname{pr}_{\mathcal{H}} \circ \mathbb{I}^{-1}: \mathcal{H} \rightarrow \mathcal{H}$.

Following [20], let us introduce the momentum

$$
\mathbf{m}=\operatorname{pr}_{\mathcal{D}} \mathbb{I} \omega+\operatorname{pr}_{\mathcal{H}} \omega=\mathbf{J} \omega, \quad \mathbf{J}=\operatorname{pr}_{\mathcal{D}} \mathbb{I}+\operatorname{pr}_{\mathcal{H}}=\mathbb{E}+\operatorname{pr}_{\mathcal{D}}(\mathbb{I}-\mathbb{E}) .
$$

Proposition 1 ([30]). The $\epsilon L R$ system on $\mathcal{M}$ has the following form:

$$
\dot{\mathbf{m}}=\epsilon[\mathbf{m}, \omega]+(1-\epsilon) \operatorname{pr}_{\mathcal{D}}[\mathbb{I} \omega, \omega], \quad \dot{\mathrm{e}}_{\mathrm{i}}=\epsilon\left[\mathrm{e}_{\mathrm{i}}, \omega\right], \quad \mathrm{i}=1, \ldots, \mathrm{n} .
$$

In the case $\epsilon=1$, the first equation in (2.10) reduces to the momentum equation (2.7) of [20]. Also, as in Theorem 3.2 [20], we have

$$
\operatorname{det} \mathbb{I} \cdot \operatorname{det} \mathbb{A}=\operatorname{det} \mathbb{I} \cdot \operatorname{det}\left(\left.\mathbb{I}^{-1}\right|_{\mathcal{H}}\right)=\operatorname{det} \mathbf{J}=\operatorname{det}\left(\left.\mathbb{I}\right|_{\mathcal{D}}\right),
$$

where $\left.\mathbb{I}\right|_{\mathcal{D}}=\operatorname{pr}_{\mathcal{D}} \circ \mathbb{I}: \mathcal{D} \rightarrow \mathcal{D}$. Therefore,

$$
\begin{aligned}
\mu_{\epsilon} \mathrm{d} \omega & =\mu_{\epsilon} \operatorname{det} \frac{\partial \omega}{\partial \mathbf{m}} \mathrm{d} \mathbf{m} \\
& =\left(\operatorname{det}\left(\left.\mathbb{I}^{-1}\right|_{\mathcal{H}}\right)\right)^{\frac{1}{2 \epsilon}}(\operatorname{det} \mathbf{J})^{-1} \mathrm{~d} \mathbf{m} \\
& =(\operatorname{det} \mathbb{I})^{-\frac{1}{2 \epsilon}}\left(\left.\operatorname{det} \mathbb{I}\right|_{\mathcal{D}}\right)^{\frac{1}{2 \epsilon}-1} \mathrm{~d} \mathbf{m} .
\end{aligned}
$$

Combining the expression of the invariant measure (2.8) and the above identity, we get the following statement: 
Theorem 2 ([30]). The $\epsilon L R$ system (2.10) has an invariant measure

$$
\left.\widetilde{\mu}_{\epsilon} \mathrm{d} \mathbf{m} \wedge \mathrm{d} e_{1} \wedge \ldots \wedge \mathrm{d} e_{n}\right|_{\mathcal{M}},
$$

where the density is given by

$$
\widetilde{\mu}_{\epsilon}=\left(\left.\operatorname{det} \mathbb{I}\right|_{\mathcal{D}}\right)^{\frac{1}{2 \epsilon}-1}=\left(\operatorname{det}\left(\left\langle\mathbb{I} e_{i}, e_{j}\right\rangle\right)\right)^{\frac{1}{2 \epsilon}-1}, \quad i, j=k+1, \ldots, n .
$$

\section{Rolling of a ball over a sphere in $\mathbb{R}^{n}$}

1. Definition of the system. We consider the Chaplygin ball type problem of rolling without slipping and twisting of an $n$-dimensional ball of radius $\rho$ in the following nonholonomic problems (see [33]):

(i) rolling over the outer surface of the $(n-1)$-dimensional fixed sphere of radius $\sigma$ (see Fig. 1 );

(ii) rolling over the inner surface of the $(n-1)$-dimensional fixed sphere of radius $\sigma, \sigma>\rho$ (see Fig. 2);

(iii) rolling over the outer surface of the $(n-1)$-dimensional fixed sphere of radius $\sigma$, but the fixed sphere is within the rolling ball $(\sigma<\rho$, in this case, the rolling ball is actually a spherical shell, see Fig. 2).

Consider the space frame $\mathbb{R}^{n}(\mathbf{x})$ with the origin $O$ at the center of the fixed sphere and the moving frame $\mathbb{R}^{n}(\mathbf{X})$ with the origin $C$ at the center of the rolling ball. The mapping from the moving frame to the space frame is given by $\mathbf{x}=g \mathbf{X}+\mathbf{r}$, where $g \in S O(n)$ is a rotation matrix and $\mathbf{r}=\overrightarrow{O C}$ is the position vector of the ball center $C$ in the space frame (see Fig. 1). The configuration space $Q$ is the direct product of the Lie group $S O(n)$ and the sphere $\mathcal{S}=\left\{\mathbf{r} \in \mathbb{R}^{n} \mid(\mathbf{r}, \mathbf{r})=(\sigma \pm \rho)^{2}\right\}$, where we take "+" for the case (i) and "-" for the cases (ii) and (iii).

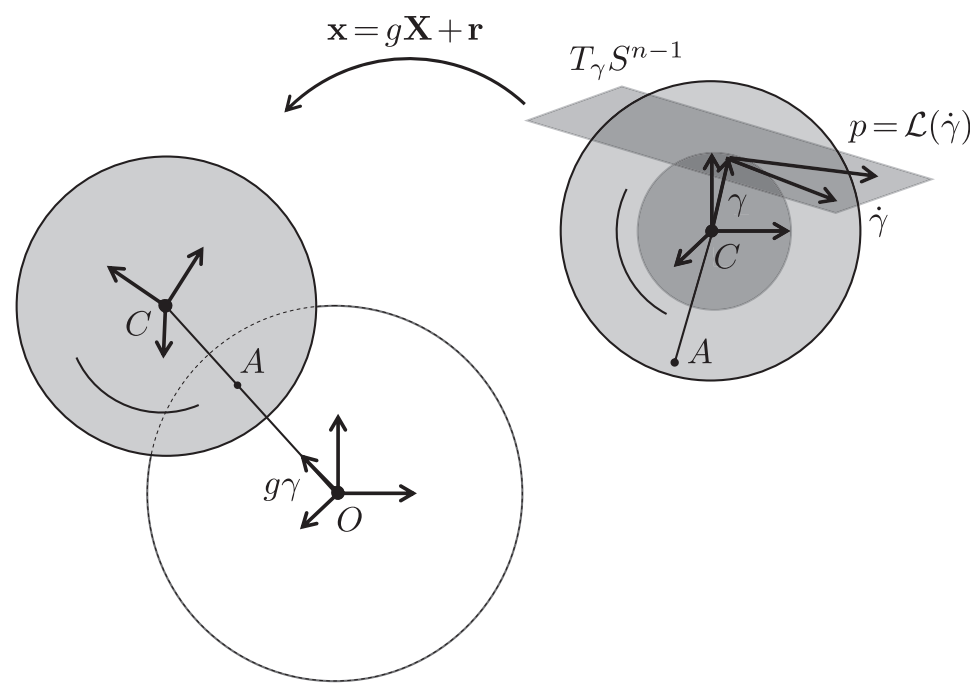

Fig. 1. The rolling without slipping and twisting of an $n$-dimensional ball of radius $\rho$ over the outer surface of the $(n-1)$-dimensional fixed sphere of radius $\sigma$ (the case (i)). $O, C$, and $A$ denote the center of the fixed sphere, the center of the rolling ball, and the contact point, respectively. 
We additionally assume that the ball is balanced, i.e., the mass center $C$ coincides with the geometrical center. Then the Lagrangian of the system is given by

$$
L(\omega, \dot{\mathbf{r}}, g, \mathbf{r})=\frac{1}{2}\langle\mathbb{I} \omega, \omega\rangle+\frac{1}{2} m(\dot{\mathbf{r}}, \dot{\mathbf{r}}),
$$

where $(\cdot, \cdot)$ is the Euclidean scalar product in $\mathbb{R}^{n},\langle\cdot, \cdot\rangle=-\frac{1}{2} \operatorname{tr}(\cdot \circ \cdot)$ is proportional to the Killing form on $s o(n), \omega=g^{-1} \dot{g}$ is the angular velocity of the ball in the moving frame, $m$ is the mass of the ball, and $\mathbb{I}: s o(n) \rightarrow s o(n)$ is the inertia operator. We use $\langle\cdot, \cdot\rangle$ to identify $s o(n)$ and $s o(n)^{*}$.

2. Dynamics and an invariant measure. Let $\Gamma=\overrightarrow{O A} /|\overrightarrow{O A}|=\frac{1}{\sigma \pm \rho} \mathbf{r}$ be the direction of the contact point $A$ in the space frame, and $\gamma=g^{-1} \Gamma$ be the direction in the frame attached to the ball (see Fig. 1). The latter is invariant under the diagonal left $S O(n)$-action:

$$
a \cdot(\omega, \dot{\mathbf{r}}, g, \mathbf{r})=(\omega, a \dot{\mathbf{r}}, a g, a \mathbf{r}), \quad a \in S O(n) .
$$

The action defines the $S O(n)$-bundle

$$
S O(n) \longrightarrow Q=S O(n) \times \mathcal{S} \stackrel{\pi}{\longrightarrow} S^{n-1}=Q / S O(n)
$$

with the submersion $\pi$ given by $\gamma=\pi(g, \mathbf{r})$.

The contact point $A$ of the ball in the moving frame is $\mathbf{X}_{A}=-( \pm \rho \gamma)$. The condition that the ball is rolling without slipping is that the velocity $\dot{\mathbf{x}}_{A}$ of the contact point in the space frame is equal to zero

$$
0=\dot{\mathbf{x}}_{A}=\frac{d}{d t}\left(g \mathbf{X}_{A}+\mathbf{r}\right)=\mp \rho \dot{g} \gamma+\dot{\mathbf{r}}=\mp \rho\left(\dot{g} g^{-1}\right) g \gamma+\dot{\mathbf{r}}
$$

$\left(\dot{\mathbf{X}}_{A}=0\right)$. This leads to the constraint

$$
\dot{\mathbf{r}}= \pm \frac{\rho}{\sigma \pm \rho} \Omega \mathbf{r}, \quad \text { i.e., } \quad \dot{\Gamma}=\rho \Omega \Gamma,
$$

where $\Omega=\operatorname{Ad}_{g} \omega=\dot{g} g^{-1}$ is the angular velocity in the space frame. From the constraint, we get the kinematic equation for $\gamma$

$$
\dot{\gamma}=\frac{d}{d t}\left(g^{-1}\right) \boldsymbol{\Gamma}+g^{-1} \dot{\boldsymbol{\Gamma}}=-g^{-1} \dot{g} g^{-1} \boldsymbol{\Gamma} \pm \frac{\rho}{\sigma \pm \rho} g^{-1} \Omega \boldsymbol{\Gamma}=-\omega \gamma \pm \frac{\rho}{\sigma \pm \rho} \omega \gamma .
$$

By introducing parameter (1.2) we can write it as a $\epsilon$-modified Poisson equation

$$
\dot{\gamma}=-\epsilon \omega \gamma \text {. }
$$

It is convenient to use orthogonal projections $\operatorname{pr}_{\mathfrak{v}_{\gamma}}$ and $\operatorname{pr}_{\mathfrak{h}_{\gamma}}$ to complementary subspaces $\mathfrak{v}_{\gamma}=\gamma \wedge \mathbb{R}^{n}$ and $\mathfrak{h}_{\gamma}=\left(\mathfrak{v}_{\gamma}\right)^{\perp}$, given by

$$
\xi \longmapsto(\xi \gamma) \wedge \gamma=\xi \gamma \otimes \gamma+\gamma \otimes \gamma \xi, \quad \text { and } \quad \xi \longmapsto \xi-(\xi \gamma) \wedge \gamma
$$

respectively. Then we have

$$
\frac{d}{d t} \operatorname{pr}_{\mathfrak{v}_{\gamma}}=\epsilon\left[\operatorname{pr}_{\mathfrak{v}_{\gamma}}, \operatorname{ad}_{\omega}\right], \quad \frac{d}{d t} \operatorname{pr}_{\mathfrak{h}_{\gamma}}=\epsilon\left[\operatorname{pr}_{\mathfrak{h}_{\gamma}}, \operatorname{ad}_{\omega}\right]
$$

where $[\cdot, \cdot]$ is the standard Lie bracket in the space of linear operators of $s o(n)$. 
No twisting at the contact point can be written as $\Omega \in \mathbf{r} \wedge \mathbb{R}^{n}$, e.g., $\omega \in \gamma \wedge \mathbb{R}^{n}$ (the angular velocity matrix $\omega$ has rank 2 and the corresponding admissible plane of rotation contains the normal vector $\gamma$ to the rolling ball at the contact point). Therefore, the constraints determine the $(n-1)$-dimensional constraint distribution

$$
\mathcal{D}=\left\{(\omega, \dot{\mathbf{r}}, g, \mathbf{r}) \mid \dot{\mathbf{r}}= \pm \frac{\rho}{\sigma \pm \rho} \operatorname{Ad}_{g}(\omega) \mathbf{r}, \operatorname{pr}_{\mathfrak{h}_{\gamma}} \omega=0\right\}
$$

which is a principal connection of the bundle (3.3). The Lagrangian is $S O(n)$-invariant as well. Thus, it is a $S O(n)$-Chaplygin system and reduces to the tangent bundle $T S^{n-1} \cong \mathcal{D} / S O(n)$.

Firstly, we will write the equations of the nonreduced problem. After imposing the constraint (3.4), we have a natural phase space of the problem

$$
\mathcal{G}=\left\{(\omega, g, \gamma) \in T S O(n) \times S^{n-1} \mid \operatorname{pr}_{\mathfrak{h}_{\gamma}} \omega=0\right\} .
$$

We define the modified inertia operator

$$
\mathbf{I}=\mathbb{I}+D \mathbb{E},
$$

where $\mathbb{E}$ is the identity operator on $s o(n)$ and $D=m \rho^{2}$. Let $m=\mathbf{I} \omega$. The equations of a motion in the variables $(\omega, g, \gamma)$ read

$$
\dot{m}=[m, \omega]+\Lambda, \quad \dot{g}=g \cdot \omega, \quad \dot{\gamma}=-\epsilon \omega \gamma .
$$

The Lagrange multiplier $\Lambda \in \mathfrak{h}_{\gamma}$ is determined from the condition that the angular velocity $\omega$ satisfies the no-twisting constraint $\omega \in \mathfrak{v}_{\gamma}=\gamma \wedge \mathbb{R}^{n}$.

The reduction of the left $S O(n)$-symmetry (3.2) induces a system on the space $\mathcal{G}_{0}=$ $=\mathcal{G} / S O(n)=\left\{(\omega, \gamma) \in s o(n) \times S^{n-1} \mid \operatorname{pr}_{\mathfrak{h}_{\gamma}} \omega=0\right\}$ given by

$$
\dot{m}=[m, \omega]+\Lambda, \quad \dot{\gamma}=-\epsilon \omega \gamma .
$$

The proof of the next theorem follows from the expression (2.7) for the modified LR system (see [33]). Let $\Omega$ be the standard invariant measure on $T S O(n)$.

Theorem 3 ([33]). The problem of the rubber rolling of a ball over a sphere (3.7) and the reduced system (3.8) possess invariant measures

$$
\left.\mu_{\epsilon}(\gamma) \Omega \wedge \mathrm{d} \gamma\right|_{\mathcal{G}},\left.\quad \mu_{\epsilon}(\gamma) \mathrm{d} \omega \wedge \mathrm{d} \gamma\right|_{\mathcal{G}_{0}}
$$

respectively, where the density $\mu_{\epsilon}(\gamma)$ is given by

$$
\mu_{\epsilon}(\gamma)=\left(\operatorname{det} \mathbf{I}^{-1} \mid \mathfrak{h}_{\gamma}\right)^{\frac{1}{2 \epsilon}}
$$

3. Momentum equation. Next, by introducing the momentum

$$
\mathbf{m}=\operatorname{pr}_{\mathfrak{v}_{\gamma}} \mathbf{I} \omega+\operatorname{pr}_{\mathfrak{h}_{\gamma}} \omega=\omega+\gamma \otimes \gamma(\mathbf{I} \omega-\omega)+(\mathbf{I} \omega-\omega) \gamma \otimes \gamma,
$$

we can describe the system (3.8) in the form

$$
\dot{\mathbf{m}}=\epsilon[\mathbf{m}, \omega]+(1-\epsilon) \operatorname{pr}_{\mathfrak{v}_{\gamma}}[\mathbf{I} \omega, \omega], \quad \dot{\gamma}=-\epsilon \omega \gamma .
$$

It can be considered also on the whole space $s o(n) \times S^{n-1}(\omega, \gamma)$, or equivalently on the space $s o(n) \times S^{n-1}(\mathbf{m}, \gamma)$. According to Theorem 2, it leads to the dual expression for an invariant measure.

Theorem 4 ([33]). The extended system (3.11) in variables $(\mathbf{m}, \gamma)$ has an invariant measure $\tilde{\mu}_{\epsilon} \mathrm{d} \mathbf{m} \wedge \mathrm{d} \gamma$,

$$
\widetilde{\mu}_{\epsilon}(\gamma)=\left(\left.\operatorname{det} \mathbf{I}\right|_{\mathfrak{v}_{\gamma}}\right)^{\frac{1}{2 \epsilon}-1}
$$




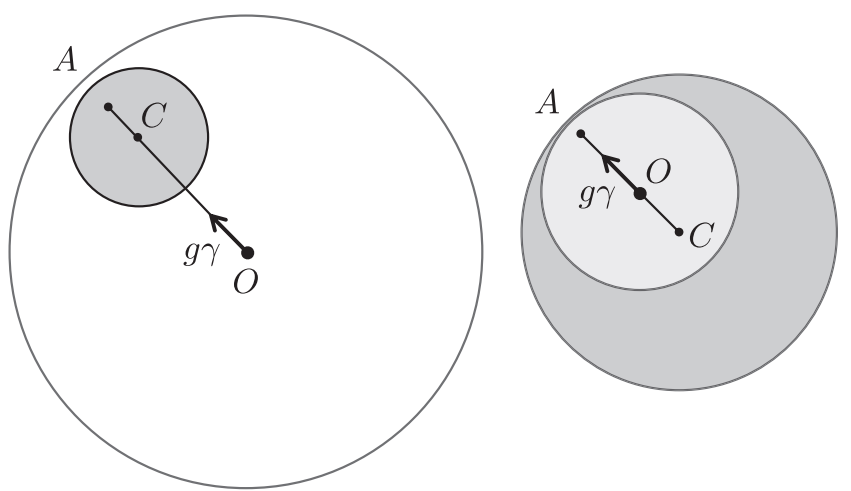

Fig. 2. The cases (ii) and (iii) in the space frame: the rolling of a ball over the inner surface of the sphere and the rolling of a spherical shell over the outer surface of the fixed sphere.

4. The reduced Lagrange-d'Alembert equation. As we have already mentioned, the system reduces to the tangent bundle $T S^{n-1} \cong \mathcal{D} / S O(n)$. The procedure of the Chaplygin reduction for rubber rolling over a sphere for $n=3$ is given by Ehlers and Koiller [17].

Recall that the vectors in $\mathcal{D}_{(g, \mathbf{r})}$ are called horizontal, while the vectors in ker $d \pi_{(g, \mathbf{r})}$ vertical. The horizontal lift $\dot{\gamma}^{h}$ of the base vector $\dot{\gamma} \in T_{\gamma} S^{n-1}$ to the horizontal space $\mathcal{D}$ at the point $(g, \mathbf{r}) \in \pi^{-1}(\gamma)$ is the unique vector in $\mathcal{D}_{(g, \mathbf{r})}$ satisfying $d \pi\left(\gamma^{h}\right)=\dot{\gamma}$. The horizontal lift $\left.\dot{\gamma}^{h}\right|_{(g, \mathbf{r})}=(\omega, \dot{\mathbf{r}})$ of $\dot{\gamma}$ is given by

$$
\begin{aligned}
\omega & =\frac{1}{\epsilon} \gamma \wedge \dot{\gamma}=\frac{\sigma \pm \rho}{\sigma} \gamma \wedge \dot{\gamma}, \\
\dot{\mathbf{r}} & =(\sigma \pm \rho) \frac{d}{d t}(g \gamma)=(\sigma \pm \rho)(\dot{g} \gamma+g \dot{\gamma})=(\sigma \pm \rho)\left(g \frac{1}{\epsilon}(\gamma \wedge \dot{\gamma}) \gamma+g \dot{\gamma}\right) \\
& =(\sigma \pm \rho)\left(1-\frac{1}{\epsilon}\right) g \dot{\gamma}=-(\sigma \pm \rho)\left( \pm \frac{\rho}{\sigma}\right) g \dot{\gamma} .
\end{aligned}
$$

Therefore, the reduced Lagrangian is

$$
\left.L_{r e d}=L\left(\left.\dot{\gamma}^{h}\right|_{(g, \mathbf{r})}, g, \mathbf{r}\right)\right)_{(g, \mathbf{r}) \in \pi^{-1}(\gamma)}=\frac{1}{2 \epsilon^{2}}\langle\mathbf{I}(\gamma \wedge \dot{\gamma}), \gamma \wedge \dot{\gamma}\rangle=-\frac{1}{2 \epsilon^{2}}(\mathbf{I}(\gamma \wedge \dot{\gamma}) \gamma, \dot{\gamma}) .
$$

The reduced Lagrange-d'Alembert equations take the form (1.1), where a $(0,3)$ tensor field $\Sigma$ depending of the curvature of connection $\mathcal{D}$ is given by (see [33])

$$
\Sigma_{\gamma}(X, Y, Z)=\frac{2 \epsilon-1}{2 \epsilon^{3}} \operatorname{tr}(\mathbf{I}(\gamma \wedge X) \circ(Y \wedge Z))=\frac{2 \epsilon-1}{\epsilon^{3}}(\mathbf{I}(\gamma \wedge X) Y, Z) .
$$

As a result, we obtain the following statement.

Theorem 5 ([33]). The Lagrange-d'Alembert equations describing the motion of the reduced system are given by

$$
\left(\epsilon \frac{d}{d t}(\mathbf{I}(\gamma \wedge \dot{\gamma}) \gamma)+(1-\epsilon) \mathbf{I}(\gamma \wedge \dot{\gamma}) \dot{\gamma}, \xi\right)=0, \quad \xi \in T_{\gamma} S^{n-1}
$$

Note that when the radii of the sphere and the ball are equal $(\epsilon=1 / 2)$, the curvature of $\mathcal{D}$ vanishes and $\Sigma \equiv 0$ - the reduced system is Hamiltonian without a time reparameterization (for $n=3$ see $[10,17]$ ). Also, if $\mathbb{I}$ is proportional to the identity operator, then $\Sigma \equiv 0$ (the reaction forces vanish, although the curvature of $\mathcal{D}$ is different from zero). 
5. Almost-Hamiltonian formulation. Consider the Legendre transformation

$$
\mathcal{L}: \quad p=\frac{\partial L_{r e d}}{\partial \dot{\gamma}}=-\frac{1}{\epsilon^{2}} \mathbf{I}(\gamma \wedge \dot{\gamma}) \gamma
$$

The point $(p, \gamma)$ belongs to the cotangent bundle of a sphere realized as a symplectic submanifold in the symplectic linear space $\left(\mathbb{R}^{2 n}(p, \gamma), d p_{1} \wedge d \gamma_{1}+\ldots+d p_{n} \wedge d \gamma_{n}\right)$ :

$$
(\gamma, \gamma)=1, \quad(\gamma, p)=0
$$

Note that here we have identified the tangent bundle $T S^{n-1}$ and the cotangent bundle $T^{*} S^{n-1}$ by means of the Euclidean metric in $\mathbb{R}^{n}$ (see Fig. 1).

The reduced flow on $T^{*} S^{n-1}$ takes the form

$$
\dot{\gamma}=X_{\gamma}(p, \gamma), \quad \dot{p}=X_{p}(p, \gamma),
$$

where $X_{\gamma}=\mathcal{L}^{-1}(p)$ is the inverse of the Legendre transformation (3.14) and

$$
X_{p}=\frac{(1-\epsilon)}{\epsilon^{3}}\left(\mathbf{I}\left(\gamma \wedge X_{\gamma}\right)\right) X_{\gamma}+\frac{(\epsilon-1)}{\epsilon^{3}}\left(\left(\mathbf{I}\left(\gamma \wedge X_{\gamma}\right)\right) X_{\gamma}, \gamma\right) \gamma-2 H \gamma
$$

(see [33]). Here

$$
H(p, \gamma)=(p, \dot{\gamma})-\left.L(\dot{\gamma}, \gamma)\right|_{\dot{\gamma}=X_{\gamma}}=\frac{1}{2}\left(X_{\gamma}, p\right)
$$

is the Hamiltonian function of the system.

Let $\mathbf{w}$ be the canonical symplectic form on $T^{*} S^{n-1}$ :

$$
\mathbf{w}=d p_{1} \wedge d \gamma_{1}+\ldots+\left.d p_{n} \wedge d \gamma_{n}\right|_{T^{*} S^{n-1}} .
$$

At the points of $T^{*} S^{n-1}$, the system (3.16) can be written in the almost Hamiltonian form

$$
\dot{x}=X_{H}=\left(X_{p}, X_{\gamma}\right), \quad i_{X_{H}}(\mathbf{w}+\sigma)=-d H,
$$

where $\sigma$ is a semibasic perturbation term determined by the tensor $\Sigma$ (see, e.g, [12, 16, 36]). The form $\mathbf{w}+\sigma$ is nondegenerate, but, in general, it is not closed.

6. The momentum equation and an invariant measure. Alternatively, the reduced equation (3.17) can be derived by using the momentum equation (3.11), since, after the reduction to the sphere $S^{n-1}$, we have

$$
\mathbf{m}=\left.\frac{1}{\epsilon}(\mathbf{I}(\gamma \wedge \dot{\gamma}) \gamma) \wedge \gamma\right|_{\dot{\gamma}=X_{\gamma}(p, \gamma)}=\epsilon \gamma \wedge p .
$$

Also, the mapping

$$
\Phi:(\gamma, p) \mapsto(\gamma, \mathbf{m}), \quad \mathbf{m}=\epsilon \gamma \wedge p,
$$

together with $\omega=\frac{1}{\epsilon} \gamma \wedge \dot{\gamma}$, maps the reduced system (3.16) to the subsystem of (3.11), and the pull-back $\Phi^{*}(\mathrm{~d} \mathbf{m} \wedge \mathrm{d} \gamma)$ is the standard volume form $\mathbf{w}^{n-1}$ on $T^{*} S^{n-1}$ (up to the multiplication by a constant). Now, from Theorem 4 , we get the following statement.

Theorem 6 ([33]). The reduced equations (3.16) has an invariant measure

$$
\left(\left.\operatorname{det} \mathbf{I}\right|_{\mathfrak{v}_{\gamma}}\right)^{\frac{1}{2 \epsilon}-1} \mathbf{w}^{n-1} \text {. }
$$




\section{Hamiltonization and integrability for a special inertia operator}

1. Chaplygin Hamiltonization. The Chaplygin multiplier is a nonvanishing function $\nu$ such that $\widetilde{\mathbf{w}}=\nu(\mathbf{w}+\sigma)$ is closed. Then the Hamiltonian vector field $\widetilde{X}_{H}$ of the function $H$ on $\left(T^{*} S^{n-1}, \widetilde{\mathbf{w}}\right)$ is proportional to the original vector field:

$$
\widetilde{X}_{H}=\frac{1}{\nu} X_{H}, \quad i_{\widetilde{X}_{H}} \widetilde{\mathbf{w}}=-d H .
$$

Thus, after the time reparameterization $d \tau=\nu d t$, the system (3.19) becomes the Hamiltonian system

$$
\frac{d}{d \tau} x=\widetilde{X}_{H}
$$

On the other hand, a classical way to introduce the Chaplygin reducing multiplier for our system is as follows (e.g., see $[14,20]$ ). Consider the time reparameterization $d \tau=\nu(\gamma) d t$, and denote $\gamma^{\prime}=d \gamma / d \tau=\dot{\gamma} / \nu$. Then the Lagrangian function transforms to $L^{*}\left(\gamma^{\prime}, \gamma\right)=L_{r e d}\left(\nu \gamma^{\prime}, \gamma\right)$. The factor $\nu$ is a Chaplygin reducing multiplier if in the new time $\tau$, the reduced system (3.13) transforms into the usual Euler - Lagrange equations of $L^{*}\left(\gamma^{\prime}, \gamma\right)$.

To relate both descriptions, define the new momenta $\widetilde{p}=\partial L^{*} / \partial \gamma^{\prime}=\nu p$. Then $\nu$ is a Chaplygin multiplier if in the new time Eqs. (3.16) become Hamiltonian with respect to the symplectic form

$$
\begin{aligned}
\widetilde{\mathbf{w}} & =d \widetilde{p}_{1} \wedge d \gamma_{1}+\ldots+\left.d \widetilde{p}_{n} \wedge d \gamma_{n}\right|_{T^{*} S^{n-1}} \\
& =\nu \mathbf{w}+\left.d \nu \wedge\left(p_{1} d \gamma_{1}+\ldots+p_{n} d \gamma_{n}\right)\right|_{T^{*} S^{n-1}}
\end{aligned}
$$

(e.g., see $[3,12,14,20,25])$. In other words,

$$
\nu \sigma=\left.d \nu \wedge\left(p_{1} d \gamma_{1}+\ldots+p_{n} d \gamma_{n}\right)\right|_{T^{*} S^{n-1}} .
$$

Here we consider a special choice of an inertia operator

$$
\mathbb{I}\left(E_{i} \wedge E_{j}\right)=\left(a_{i} a_{j}-D\right) E_{i} \wedge E_{j} \quad \text { i.e., } \quad \mathbf{I}(X \wedge Y)=A X \wedge A Y,
$$

where $A=\operatorname{diag}\left(a_{1}, \ldots, a_{n}\right)$, when the density (3.12) significantly simplifies, and it is proportional to $($ see $[20,30])$

$$
(\gamma, A \gamma)^{\left(\frac{1}{2 \epsilon}-1\right)(n-2)}
$$

The reduced Lagrangian $L_{r e d}$ and the reduced equations take the form

$$
\begin{aligned}
& L_{r e d}=\frac{1}{2 \epsilon^{2}}\left((A \dot{\gamma}, \dot{\gamma})(A \gamma, \gamma)-(A \gamma, \dot{\gamma})^{2}\right), \\
& \epsilon \frac{d}{d t}((A \gamma, \dot{\gamma}) A \gamma-(A \gamma, \gamma) A \dot{\gamma})=(\epsilon-1)((A \dot{\gamma}, \dot{\gamma}) A \gamma-(A \gamma, \dot{\gamma}) A \dot{\gamma})+\lambda \gamma,
\end{aligned}
$$

respectively. The Lagrange multiplier $\lambda$ is determined by the condition that a trajectory $\gamma(t)$ belongs to the sphere $(\gamma, \gamma)=1$.

The existence of a Chaplygin reducing multiplier $\nu(\gamma)$ implies that the original system has an invariant measure $\nu^{n-2} \mathbf{w}^{n-1}$ (e.g., see Theorem 3.5, [20]). From the expression of the density (4.2), we get that a possible Chaplygin multiplier should be proportional to $(A \gamma, \gamma)^{\frac{1}{2 \epsilon}-1}$. Indeed, we have: 
Theorem 7 ([33]). After the time reparameterization $d \tau=\nu(\gamma)=\epsilon(A \gamma, \gamma)^{\frac{1}{2 \epsilon}-1} d t$, the reduced system (4.4) becomes the geodesic flow of the metric defined by the Lagrangian

$$
L^{*}\left(\gamma^{\prime}, \gamma\right)=\frac{1}{2}(\gamma, A \gamma)^{\frac{1}{\epsilon}-2}\left(\left(A \gamma^{\prime}, \gamma^{\prime}\right)(A \gamma, \gamma)-\left(A \gamma, \gamma^{\prime}\right)^{2}\right)
$$

Let us mention that in the three-dimensional case the system is always Hamiltonizable due to the fact that it has an invariant measure and that the reduced configuration space is 2-dimensional. For $n=3$ all inertia operators can be written in the form (4.1) and Theorem 7 reduces to the one given in [17], where the procedure of reduction for rubber rolling over a sphere for $n=3$ is given.

2. Integrability for $\boldsymbol{\rho}=\mathbf{2} \boldsymbol{\sigma}$. In [9], for $n=3$, Borisov and Mamaev proved the integrability of the rubber rolling for a specific ratio between the radii of the ball and the spherical shell $\rho=2 \sigma$, i.e, $\epsilon=-1$.

In the new coordinates

$$
x=\frac{A^{\frac{1}{2}} \gamma}{\sqrt{(A \gamma, \gamma)}}
$$

the Lagrangian (4.5) transforms to $L^{*}\left(x^{\prime}, x\right)=\frac{1}{2}\left(x, A^{-1} x\right)^{-\frac{1}{\epsilon}}\left(x^{\prime}, x^{\prime}\right)$ on the sphere $(x, x)=1$. Together with $L^{*}\left(x^{\prime}, x\right)$, we consider a natural mechanical system with the Lagrangian

$$
L_{\epsilon}=\frac{1}{2}\left(\frac{d x}{d s}, \frac{d x}{d s}\right)-V_{\epsilon}(x), \quad V_{\epsilon}(x)=-\left(A^{-1} x, x\right)^{-\frac{1}{\epsilon}} .
$$

Using the Maupertius principle and the Chaplygin time reparameterization one gets:

Proposition 2 ([24]). The trajectories $\gamma(t)$ of the rolling of a rubber Chaplygin ball over a spherical surface determined by Eq. (4.4), with the unit kinetic energy $L_{\text {red }}(\dot{\gamma}, \gamma)=1$, under the transformation (4.6) and a time reparameterization

$$
d s=\epsilon\left(A^{-1} x, x\right)^{1+\frac{1}{2 \epsilon}} d t \quad\left(=\epsilon(A \gamma, \gamma)^{-1-\frac{1}{2 \epsilon}} d t\right),
$$

are mapped to the zero-energy trajectories $x(s)$ of the natural mechanical systems with the Lagrangian $L_{\epsilon}$ :

$$
\frac{d^{2}}{d s^{2}} x=-\frac{2}{\epsilon}\left(A^{-1} x, x\right)^{-\frac{1}{\epsilon}-1} A^{-1} x+\lambda x, \quad \lambda=\frac{2}{\epsilon}\left(A^{-1} x, x\right)^{-\frac{1}{\epsilon}}-\left(\frac{d x}{d s}, \frac{d x}{d s}\right) .
$$

Among the potentials $V_{\epsilon}$, there are two exceptional ones determining completely integrable systems: for $\epsilon=+1$ we have Braden's [11] and for $\epsilon=-1$ Neumann's potential [35].

Theorem 8 ([24]). For an inertia operator (4.1) and $\rho=2 \sigma(\epsilon=-1)$, the reduced problem of the rolling of a rubber Chaplygin ball over a spherical surface is completely integrable: under the transformation (4.6) and a time reparameterization

$$
d s=-\left(A^{-1} x, x\right)^{\frac{1}{2}} d t \quad\left(=-(A \gamma, \gamma)^{-\frac{1}{2}} d t\right),
$$

the solutions $\gamma(t)$ of (4.4) with the unit kinetic energy $L_{\text {red }}(\dot{\gamma}, \gamma)=1$ are mapped to the zeroenergy trajectories $x(s)$ of the Neumann system with Lagrangian $L_{-1}$.

RUSSIAN JOURNAL OF NONLINEAR DYNAMICS, 2019, 15(4), 457-475 
Equation (4.4) for $\epsilon=1$,

$$
\frac{d}{d t}((A \gamma, \dot{\gamma}) A \gamma-(A \gamma, \gamma) A \dot{\gamma})=\lambda \gamma
$$

coincides with the reduced equation of the nonholonomic Veselova problem studied in [20]. In the 3 -dimensional case the paper [40] established a relation between the Veselova problem and the Neumann system. This result was generalized in [20] as follows. Under a time reparameterization

$$
d s_{1}=\sqrt{\frac{(A \dot{\gamma}, \dot{\gamma})(A \gamma, \gamma)-(A \gamma, \dot{\gamma})^{2}}{(A \gamma, \gamma)}} d t
$$

the solutions $x(t)=\gamma(t)$ of (4.8) transform to solutions of the Neumann problem with the potential $V(x)=\frac{1}{2}\left(A^{-1} x, x\right)$,

$$
\frac{d^{2}}{d s_{1}^{2}} x=-A^{-1} x+\lambda x, \quad \lambda=\left(A^{-1} x, x\right)-\left(\frac{d x}{d s_{1}}, \frac{d x}{d s_{1}}\right),
$$

which belong to the invariant set $\left(A \frac{d x}{d s_{1}}, \frac{d x}{d s_{1}}\right)(A x, x)-\left(A x, \frac{d x}{d s_{1}}\right)^{2}-(A x, x)=0$. Proposition 2 gives another trajectory isomorphism.

Theorem 9 ([24]). Under the transformation (4.6) and a time reparameterization $d s=$ $=\left(A^{-1} x, x\right)^{\frac{3}{2}} d t$, the unit kinetic energy trajectories $\gamma(t)$ of (4.8) are mapped to the zero-energy trajectories $x(s)$ of the Braden system with the Lagrangian $L_{+1}$.

3. Separation of variables in the case $\boldsymbol{\rho}=\mathbf{2} \boldsymbol{\sigma}$. Let us assume $a_{1}>a_{2}>\ldots>a_{n}>0$ and $\epsilon=-1$. In the three-dimensional case Borisov and Mamaev constructed separating variables of the system as a deformation of sphero-conical coordinates [9]. A similar type of deformations are used in $[8,37,38]$, where they are called nonholonomic deformations of sphero-conical coordinates or quasi-sphero-conical coordinates. Separation variables of the Neumann system explains, in some sense, the unusual choice of variables given in [9].

For an $n$-dimensional Neumann system, the Hamilton-Jacobi equation can be solved by the separation of variables in standard sphero-conical coordinates $u_{1}, \ldots, u_{n-1}$ (see [35]). Using Theorem 8, after a time reparameterization, the rolling ball system separates in coordinates $u_{1}, \ldots, u_{n-1}$. In the case of the potential $V(x)=-\left(A^{-1} x, x\right)$ they are defined by the equations $($ see $[35])$

$$
\sum_{i=1}^{n} \frac{x_{i}^{2}}{z-a_{i}^{-1}}=\prod_{i=1}^{n-1} \frac{\left(z-u_{i}\right)}{U(z)}, \quad U(z)=\prod_{j=1}^{n}\left(z-a_{j}^{-1}\right),
$$

and $a_{1}^{-1}<u_{1}<a_{2}^{-1}<u_{2}<\ldots a_{n-1}^{-1}<u_{n-1}<a_{n}^{-1}$. Formulas for $x_{i}$ are [35]:

$$
x_{i}^{2}=\frac{\left(a_{i}^{-1}-u_{1}\right)\left(a_{i}^{-1}-u_{2}\right) \ldots\left(a_{i}^{-1}-u_{n-1}\right)}{\left(a_{i}^{-1}-a_{1}^{-1}\right)\left(a_{i}^{-1}-a_{2}^{-1}\right) \ldots\left(a_{i}^{-1}-a_{n}^{-1}\right)}, \quad i=1, \ldots, n .
$$

Therefore, one gets

$$
\gamma_{i}^{2}=\frac{\left(a_{i}^{-1}-u_{1}\right)\left(a_{i}^{-1}-u_{2}\right) \ldots\left(a_{i}^{-1}-u_{n-1}\right)}{a_{i} \nu^{2}\left(a_{i}^{-1}-a_{1}^{-1}\right)\left(a_{i}^{-1}-a_{2}^{-1}\right) \ldots\left(a_{i}^{-1}-a_{n}^{-1}\right)}, \quad i=1, \ldots, n,
$$

where $\nu^{2}=\left(A^{-1} x, x\right)=(A \gamma, \gamma)^{-1}=a_{1}^{-1}+a_{2}{ }^{-1}+\ldots+a_{n}{ }^{-1}-u_{1}-u_{2}-\ldots-u_{n-1}$. 
In the three-dimensional case, the modified operator $\mathbf{I}$ corresponds to the matrix $\mathbf{J}=\operatorname{diag}\left(J_{1}, J_{2}, J_{3}\right)$ used in [9], where $J_{1}=a_{2} a_{3}, J_{2}=a_{1} a_{3}, J_{3}=a_{1} a_{2}$. In [9] the separating coordinates $u, v$ are defined by the equation

$$
\frac{1}{\eta^{2}} \sum_{i=1}^{3} \frac{\gamma_{i}^{2}}{\left(J_{i}-z\right) J_{i}}=\frac{(z-u)(z-v)}{J_{1} J_{2} J_{3}\left(J_{1}-z\right)\left(J_{2}-z\right)\left(J_{3}-z\right)}
$$

where $0<J_{1}<u<J_{2}<v<J_{3}$, and $\eta^{2}=\left(\mathbf{J}^{-1} \gamma, \gamma\right)$.

From (4.10) it follows that [9]

$$
\begin{aligned}
& \gamma_{1}^{2}=\eta^{2} \frac{J_{1}\left(J_{1}-u\right)\left(J_{1}-v\right)}{\left(J_{1}-J_{2}\right)\left(J_{1}-J_{3}\right)}, \\
& \gamma_{2}^{2}=\eta^{2} \frac{J_{2}\left(J_{2}-u\right)\left(J_{2}-v\right)}{\left(J_{2}-J_{1}\right)\left(J_{2}-J_{3}\right)}, \\
& \gamma_{3}^{2}=\eta^{2} \frac{J_{3}\left(J_{3}-u\right)\left(J_{3}-v\right)}{\left(J_{3}-J_{1}\right)\left(J_{3}-J_{2}\right)}, \quad \eta^{2}=\left(J_{1}+J_{2}+J_{3}-u-v\right)^{-1} .
\end{aligned}
$$

Since $\eta^{2}=\left(a_{1} a_{2} a_{3}\right)^{-1} \nu^{-2}$, the expressions (4.11) and (4.9) (for $n=3$ ) coincide, where $u=a_{1} a_{2} a_{3} u_{1}$ and $v=a_{1} a_{2} a_{3} u_{2}$.

4. Noncommutative integrability of a symmetric case. For any pair of equal parameters $a_{i}=a_{j}$, the geodesic flow of the metric given by (4.5) (for any value of the parameter $\epsilon$ ) has the additional Noether integral

$$
\Phi_{i j}\left(\gamma^{\prime}, \gamma\right)=\gamma_{i} \frac{\partial L^{*}}{\partial \gamma_{j}^{\prime}}-\gamma_{j} \frac{\partial L^{*}}{\partial \gamma_{i}^{\prime}}
$$

In other words, the natural mechanical system (4.7) preserves the function

$$
\widetilde{\Phi}_{i j}\left(\frac{d x}{d s}, x\right)=x_{i} \frac{d x_{j}}{d s}-x_{j} \frac{d x_{i}}{d s} .
$$

If we have at least three equal parameters, the systems are integrable according to the noncommutative version of the Liouville theorem. More precisely, assume $\{1,2, \ldots, n\}=$ $=I_{0} \cup I_{1} \cup \ldots \cup I_{r}, I_{i} \cap I_{j}=\varnothing$,

$$
a_{i_{0}}=\alpha_{0}, i_{0} \in I_{0}, \quad a_{i_{1}}=\alpha_{1}, i_{1} \in I_{1}, \quad \ldots \quad a_{i_{r}}=\alpha_{r}, i_{r} \in I_{r}
$$

$\alpha_{i} \neq \alpha_{j}, i \neq j$. Then the geodesic flow of (4.5) and the system (4.7) are $S O\left(\left|I_{0}\right|\right) \times \ldots \times$ $S O\left(\left|I_{r}\right|\right)$-symmetric with Noether's integrals (4.12) and (4.13), respectively. For $\epsilon= \pm 1$ they are completely integrable in a noncommutative sense: the corresponding Hamiltonian flows on the cotangent bundle of a sphere $S^{n-1}$ are generically quasi-periodic over invariant isotropic tori of dimension

$$
N=r+\sharp\left\{I_{i}|| I_{i} \mid \geqslant 2\right\}
$$

(e.g, see $[15,29]$, where a detailed analysis for natural mechanical systems on a symmetric ellipsoid is given). 
5. Integrability for $\boldsymbol{\epsilon} \neq \pm \mathbf{1}$. Note that in the case of $S O(n)$-symmetry, when $A$ is proportional to the identity matrix, trajectories of (4.4) are great circles for all $\epsilon$. If the symmetry group is large enough, integrability follows from conservations of the Noether integrals and the Lagrangian (4.5). Then the restriction $\epsilon= \pm 1$ is not essential.

Let $\epsilon$ be arbitrary.

Theorem 10. For the inertia operator (4.1), where

$$
a_{1}=a_{2}=\ldots=a_{l} \neq a_{l+1}=a_{l+2}=\ldots=a_{n},
$$

the reduced system (3.13) is integrable: generic motions, up to a time reparameterization, are quasi-periodic over three dimensional invariant tori. For $l=1$ or $l=n-1$, the invariant tori are two-dimensional.

\section{Integrability for a symmetric rigid body inertia operator}

1. Rigid body inertia operator. Generally, for $n \geqslant 4$, the operator (4.1) is not a physical inertia operator of a multidimensional rigid body. Let $\varrho(\mathbf{X})$ be the density of the ball. The mass tensor $I$ is defined by $I=\int \varrho(\mathbf{X}) \mathbf{X} \otimes \mathbf{X} d \mathbf{X}$, e.g., see [21, 23]. It is diagonal in the moving orthonormal base determined by the principal axes of inertia. Then the rigid body inertia operator has the form

$$
\omega \longmapsto I \omega+\omega I, \quad I=\operatorname{diag}\left(I_{1}, \ldots, I_{n}\right) .
$$

Recently, the integrability of the Veselova problem (which can been seen as the limiting case $\epsilon=+1$ ) with a physical inertia operator (5.1), where

$$
I_{1}=I_{2}=\ldots=I_{r} \neq I_{r+1}=I_{r+2}=\ldots=I_{n},
$$

without involving Chaplygin Hamiltonization has been proved by Fasso, Garcia-Naranjo, and Montaldi [18].

Let us consider the rolling ball over a sphere for the inertia operator $\mathbb{I}$ of the form (5.1) as well. Then we can write the modified operator $\mathbf{I}=\mathbb{I}+D \cdot \mathbb{E}$ as

$$
\mathbf{I} \omega=\epsilon^{2}(J \omega+\omega J),
$$

where

$$
J=\operatorname{diag}\left(J_{1}, \ldots, J_{n}\right)=\frac{1}{\epsilon^{2}} \operatorname{diag}\left(I_{1}+\frac{D}{2}, \ldots, I_{n}+\frac{D}{2}\right),
$$

and the Legendre transformation (3.14) takes the form

$$
p=-J(\gamma \wedge \dot{\gamma}) \gamma-(\gamma \wedge \dot{\gamma}) J \gamma=J \dot{\gamma}+(J \gamma, \gamma) \dot{\gamma}-(J \gamma, \dot{\gamma}) \gamma
$$

It coincides with the Legendre transformation of the Veselova problem considered in [18] (see Proposition 3.4). That is why we can use the inverse

$$
\dot{\gamma}=X_{\gamma}(p, \gamma)=C_{\gamma}\left(p-\frac{\left(p, C_{\gamma}(\gamma)\right)}{\left(\gamma, C_{\gamma}(\gamma)\right)} \gamma\right)
$$

derived there. Here

$$
C_{\gamma}=\operatorname{diag}\left(J_{1}+(\gamma, J \gamma), \ldots, J_{n}+(\gamma, J \gamma)\right)^{-1}
$$

and (5.6) is the unique inverse of (5.5) for $p, \gamma, \dot{\gamma}$ that satisfy (3.15) and $(\dot{\gamma}, \gamma)=0$. 
From (3.17), (5.3), the equation in $p$ becomes

$$
\dot{p}=X_{p}=\frac{1-\epsilon}{\epsilon}\left(\left(X_{\gamma}, X_{\gamma}\right) J \gamma-\left(\gamma, J X_{\gamma}\right) X_{\gamma}-\left(X_{\gamma}, X_{\gamma}\right)(\gamma, J \gamma) \gamma\right)-2 H \gamma,
$$

with the Hamiltonian $H$

$$
H(p, \gamma)=\frac{1}{2}\left(p, X_{\gamma}\right)=\frac{1}{2}\left(p, C_{\gamma}(p)\right)-\frac{1}{2} \frac{\left(p, C_{\gamma}(\gamma)\right)^{2}}{\left(\gamma, C_{\gamma}(\gamma)\right)}
$$

By comparing Theorem 6 and the expression of an invariant measure of the Veselova problem given by Fasso, Garcia-Naranjo, and Montaldi (see Proposition 3.7, [18]), we get:

Theorem 11. The reduced equations of the rolling of a ball over a sphere without slipping and twisting with the rigid body inertia operator (5.1) are given by (5.6), (5.7), whereas $J$ is defined by (5.4). The reduced system has an invariant measure

$$
\mu(\gamma) \mathbf{w}^{n-1}=\left(\frac{\operatorname{det} C_{\gamma}}{\left(\gamma, C_{\gamma}(\gamma)\right)}\right)^{1-\frac{1}{2 \epsilon}} \mathbf{w}^{n-1}
$$

2. Integrals and nonexistence of a Chaplygin multiplier. Assume $I_{i}=I_{j}$, i.e., $J_{i}=J_{j}$. Then the Lagrangian $L_{r e d}$ and the Hamiltonian $H$ are invariant under rotations in the $\left(\gamma_{i}, \gamma_{j}\right)$-plane. We can expect the associated Noether type integral linear in momenta (e.g., see $[1,31])$. We have

Theorem $12([27])$. Let $J_{i}=J_{j}$. Then

$$
\phi_{i j}=\left(J_{i}+(\gamma, J \gamma)\right)^{\frac{1}{2 \epsilon}-1}\left(\gamma_{i} p_{j}-\gamma_{j} p_{i}\right) .
$$

is the first integral of the reduced flow (5.6), (5.7).

Note that the integral $\phi_{i j}$ is not the integral given by the so-called nonholonomic Noether theorem (e.g., see $[1,19,32])$, since the generator of the associated $S O(2)$-action on the configuration space $Q$ is not a section of the distribution $\mathcal{D}$.

For $\epsilon=1 / 2$ the system is already Hamiltonian.

Theorem $13([27])$. Assume that $n \geqslant 4, \epsilon \neq 1 / 2$, and $J_{i}=J_{j} \neq J_{k}=J_{l}$ for some mutually different indexes $i, j, k, l$. Then the reduced flow (5.6), (5.7) does not allow a Chaplygin multiplier.

The proof given in [27] is based on the following simple observations. Assume that $\nu$ is a Chaplygin multiplier and that and $J_{i}=J_{j} \neq J_{k}=J_{l}$ for some mutually different indexes $i, j, k, l$. From (5.9) and the fact that the system has an invariant measure $\nu^{n-2} \mathbf{w}^{n-1}$, the Hamiltonian $(5.8) H(\widetilde{p}, \gamma)=\left.H(p, \gamma)\right|_{p=\widetilde{p} / \nu}$ is invariant under rotations in the planes $\left(\widetilde{p}_{i}, \widetilde{p}_{j}, \gamma_{i}, \gamma_{j}\right)$ and $\left(\widetilde{p}_{k}, \widetilde{p}_{l}, \gamma_{k}, \gamma_{l}\right)$. Thus, the Hamiltonian flow of $H$ has the Noether integrals $\Phi_{i j}=\gamma_{i} \widetilde{p}_{j}-\gamma_{j} \widetilde{p}_{i}$ and $\Phi_{k l}=\gamma_{k} \widetilde{p}_{l}-\gamma_{l} \widetilde{p}_{k}$ (see, e.g., [1,31]), implying that the original system has the same integrals, in coordinates $(p, \gamma)$ given by $\Phi_{i j}=\nu\left(\gamma_{i} p_{j}-\gamma_{j} p_{i}\right), \Phi_{k l}=\nu\left(\gamma_{k} p_{l}-\gamma_{l} p_{k}\right)$. On the other hand, according to Theorem $12, \phi_{i j}, \phi_{k l}$ are also integrals of the motion. Since $\epsilon \neq 1 / 2$, one gets $J_{i}=J_{k}$, which is a contradiction (see [27]). 
The above considerations cannot be applied if we assume that $n-1$ parameters $I_{i}$ are equal, for example, $I_{1}=I_{2}=\ldots=I_{n-1}(S O(n-1)$-symmetric rigid body, multidimensional Lagrange top). Moreover, in that case $\mathbb{I}$ is of the form (4.1), where $A=\operatorname{diag}\left(a_{1}, \ldots, a_{1}, a_{n}\right)$ is defined by

$$
I_{1}=\ldots=I_{n-1}=\frac{a_{1}^{2}-D}{2}, \quad I_{n}=a_{1} a_{n}-\frac{a_{1}^{2}+D}{2} .
$$

Then $\epsilon(A \gamma, \gamma)^{\frac{1}{2 \epsilon}-1}$ is a Chaplygin multiplier and it is proportional to $\left(J_{1}+(\gamma, J \gamma)\right)^{\frac{1}{2 \epsilon}-1}$.

In Section 4, for $a_{i}=a_{j}$, we also used the Noether integral (4.12), which in the original coordinates $(p, \gamma)$ takes the form $\Phi_{i j}=\epsilon(A \gamma, \gamma)^{\frac{1}{2 \epsilon}-1}\left(\gamma_{i} p_{j}-\gamma_{j} p_{i}\right)$. These are the examples of the following general statement:

Lemma 1. Let $(Q, L, \mathcal{D}, G)$ be a $G$-Chaplygin system with the base manifold $N=Q / G$. Assume that the reduced almost-Hamiltonian system on $T^{*} N(p, x)$ admits a Chaplygin reducing multiplier $\nu$ and that the corresponding flow of the Hamiltonian vector field $\widetilde{X}_{H}=(-\partial H / \partial x, \partial H / \partial \widetilde{p})$ has the integral $f(\widetilde{p}, x)$. Then the original system also preserves the function $f(p, x)=\left.f(\widetilde{p}, x)\right|_{\tilde{p}=\nu p}$.

Namely, the vector field $X_{H}$ of the original system is proportional to the Hamiltonian vector field: $\widetilde{X}_{H}=\frac{1}{\nu} X_{H}$. Therefore,

$$
\frac{d}{d t} f=d f\left(X_{H}\right)=\nu d f\left(\widetilde{X}_{H}\right)=\nu \frac{d}{d \tau} f=0 .
$$

Note that we use the same notation for a function $f$ in variables $(p, x)$ and $(\widetilde{p}, x)$, having in mind that $f(p, x)$ and $f(\widetilde{p}, x)$ represent the same function on the cotangent bundle $T^{*} N$. The Noether theorem for the reduced Chaplygin systems is also studied in [26].

3. Integrability of a symmetric case. Firstly, recall that in the case of $S O(n)$-symmetry, when the mass tensor $I$ (i.e., the matrix $J$ ) is proportional to the identity matrix, the $(0,3)$-tensor $\Sigma$ vanishes and the trajectories of (5.6), (5.7) are great circles for all $\epsilon$. Similarly, as for the Veselova problem [18], we have the following statement.

Theorem 14. For the symmetric inertia operator (5.1), (5.2), the reduced system (5.6), (5.7) is solvable by quadratures and we have:

(i) If $r \neq 1, n-1$, generic motions are quasi-periodic over 3 -dimensional invariant tori that are level sets of integrals $H, \phi_{i j}, \phi_{k l}, 1 \leqslant j<i \leqslant r, r<l<k \leqslant n$.

(ii) If $r=n-1$, generic motions are quasi-periodic over 2 -dimensional invariant tori that are level sets of $H, \phi_{i j}, 1 \leqslant j<i \leqslant n-1$ (similarly for $r=1$ ).

For $\epsilon=1 / 2$ the system is Hamiltonian and the proof, as in Theorem 10, follows from the theorem on noncommutative integrability of the Hamiltonian systems. The case $r=n-1$ is similar: the function $\nu(\gamma)=\left(J_{1}+(\gamma, J \gamma)\right)^{\frac{1}{2 \epsilon}-1}$ is a Chaplygin multiplier and the integrability is established in Theorem 10. According to Theorem 13, for $\epsilon \neq 1 / 2$ and $r \neq 1, n-1$, the system does not have a Chaplygin multiplier. However, we can apply a variant of the reduction method used for the Veselova problem in [18].

Without loss of generality it can be assumed that $n=4, r=2$ [27]. Since the system is $S O(2) \times S O(2)$-invariant, we can pass to the second reduced space $P=T^{*} S^{3} / S O(2) \times S O(2)$. The regular compact connected components of invariant varieties

$$
\phi_{21}=c_{1}, \quad \phi_{43}=c_{2}, \quad H=h
$$


are 3-dimensional and $S O(2) \times S O(2)$-invariant. Therefore, the reduced invariant sets on $P$ are relative periodic orbits. Hence, we get that generic trajectories are quasi-periodic over 3 -dimensional invariant tori (for the reconstruction of relative periodic orbits, see, e.g, [18, 22]).

Thus, for $r \neq 1, n-1$ and $\epsilon \neq 1 / 2$, the problem is quasi-periodic, has an invariant measure and, according to Theorem 13, does not allow the Chaplygin reducing multiplier. Closely related, let us note that the rolling of the ball over a horizontal plane without spinning and twisting, where the mass center does not coincide with the geometrical center, provides an example of the system such that the appropriate phase space is foliated by invariant tori, but the system itself does not have an analytic invariant measure and is not Hamiltonizable either (see [3]).

\section{Acknowledgments}

We would like to thank the organizers of the conference "Scientific Heritage of Sergey A. Chaplygin: Nonholonomic Mechanics, Vortex Structures and Hydrodynamics", Cheboksary, 2-6 June, 2019, for kind hospitality.

\section{References}

[1] Arnol'd, V.I., Kozlov, V.V., and Neřshtadt, A.I., Mathematical Aspects of Classical and Celestial Mechanics, 3rd ed., Encyclopaedia Math. Sci., vol.3, Berlin: Springer, 2006.

[2] Bakša, A., On Geometrization of Some Nonholonomic Systems, Theor. Appl. Mech., 2017, vol.44, no. 2, pp. 133-140; see also: Mat. Vesnik, 1975, vol. 27, pp. 233-240.

[3] Bolsinov, A. V., Borisov, A. V., and Mamaev, I. S., Rolling of a Ball without Spinning on a Plane: The Absence of an Invariant Measure in a System with a Complete Set of Integrals, Regul. Chaotic Dyn., 2012, vol. 17, no. 6, pp. 571-579.

[4] Borisov, A. V., Mamaev, I. S., and Bizyaev, I. A., The Hierarchy of Dynamics of a Rigid Body Rolling without Slipping and Spinning on a Plane and a Sphere, Regul. Chaotic Dyn., 2013, vol.18, no. 3, pp. 277-328.

[5] Bolsinov, A. V., Borisov, A. V., and Mamaev, I. S., Geometrisation of Chaplygin's Reducing Multiplier Theorem, Nonlinearity, 2015, vol. 28, no. 7, pp. 2307-2318.

[6] Bolsinov, A.V., Complete Commutative Subalgebras in Polynomial Poisson Algebras: A Proof of the Mischenko-Fomenko Conjecture, Theor. Appl. Mech., 2016, vol. 43, no. 2, pp. 145-168.

[7] Borisov, A. V. and Fedorov, Yu. N., On Two Modified Integrable Problems in Dynamics, Mosc. Univ. Mech. Bull., 1995, vol. 50, no. 6, pp.16-18; see also: Vestnik Moskov. Univ. Ser. 1. Mat. Mekh., 1995, no. 6 , pp. 102-105.

[8] Borisov, A. V., Fedorov, Yu. N., and Mamaev, I. S., Chaplygin Ball over a Fixed Sphere: An Explicit Integration, Regul. Chaotic Dyn., 2008, vol.13, no. 6, pp. 557-571.

[9] Borisov, A. V. and Mamaev, I. S., Rolling of a Non-Homogeneous Ball over a Sphere without Slipping and Twisting, Regul. Chaotic Dyn., 2007, vol.12, no. 2, pp.153-159.

[10] Borisov, A. V., Mamaev, I. S., and Treschev, D. V., Rolling of a Rigid Body without Slipping and Spinning: Kinematics and Dynamics, J. Appl. Nonlinear Dyn., 2013, vol. 2, no. 2, pp. 161-173.

[11] Braden, H.W., A Completely Integrable Mechanical System, Lett. Math. Phys., 1982, vol.6, no.6, pp. 449-452.

[12] Cantrijn, F., Cortés, J., de León, M., and Martín de Diego, D., On the Geometry of Generalized Chaplygin Systems, Math. Proc. Cambridge Philos. Soc., 2002, vol. 132, no. 2, pp. 323-351.

[13] Chaplygin, S. A., On a Ball's Rolling on a Horizontal Plane, Regul. Chaotic Dyn., 2002, vol. 7, no. 2, pp. 131-148; see also: Math. Sb., 1903, vol.24, no.1, pp. 139-168.

RUSSIAN JOURNAL OF NONLINEAR DYNAMICS, 2019, 15(4), 457-475 
[14] Chaplygin, S.A., On the Theory of Motion of Nonholonomic Systems. The Reducing-Multiplier Theorem, Regul. Chaotic Dyn., 2008, vol.13, no.4, pp.369-376; see also: Mat. Sb., 1912, vol.28, no. 2, pp. 303-314.

[15] Davison, Ch. M., Dullin, H.R., and Bolsinov, A.V., Geodesics on the Ellipsoid and Monodromy, J. Geom. Phys., 2007, vol.57, no. 12, pp. 2437-2454.

[16] Ehlers, K., Koiller, J., Montgomery, R., and Rios, P. M., Nonholonomic Systems via Moving Frames: Cartan Equivalence and Chaplygin Hamiltonization, in The Breadth of Symplectic and Poisson Geometry, Progr. Math., vol. 232, Boston, Mass.: Birkhäuser, 2005, pp. 75-120.

[17] Koiller, J. and Ehlers, K., Rubber Rolling over a Sphere, Regul. Chaotic Dyn., 2007, vol. 12, no. 2, pp. 127-152.

[18] Fassò, F., García-Naranjo, L. C., and Montaldi, J., Integrability and Dynamics of the $n$-Dimensional Symmetric Veselova Top, J. Nonlinear Sci., 2019, vol. 29, no. 3, pp. 1205-1246.

[19] Fassò, F., García-Naranjo, L. C., and Sansonetto, N., Moving Energies As First Integrals of Nonholonomic Systems with Affine Constraints, Nonlinearity, 2018, vol. 31, no. 3, pp. 755-782.

[20] Fedorov, Yu. N. and Jovanović, B., Nonholonomic LR Systems As Generalized Chaplygin Systems with an Invariant Measure and Flows on Homogeneous Spaces, J. Nonlinear Sci., 2004, vol. 14, no. 4, pp. 341-381.

[21] Fedorov, Yu. N. and Kozlov, V. V., Various Aspects of $n$ frm[o]-Dimensional Rigid Body Dynamics, Amer. Math. Soc. Transl. Ser. 2, 1995, vol.168, pp. 141-171.

[22] Field, M. J., Equivariant Dynamical Systems, Trans. Amer. Math. Soc., 1980, vol. 259, no. 1, pp. 185205.

[23] Gajić, B., The Rigid Body Dynamics: Classical and Algebro-Geometric Integration, Zb. Rad. (Beogr.), 2013, vol. 16(24), pp. 5-44.

[24] Gajić, B. and Jovanović, B., Nonholonomic Connections, Time Reparametrizations, and Integrability of the Rolling Ball over a Sphere, Nonlinearity, 2019, vol. 32, no. 5, pp. 1675-1694.

[25] García-Naranjo, L. C., Generalisation of Chaplygin's Reducing Multiplier Theorem with an Application to Multi-Dimensional Nonholonomic Dynamics, J. Phys. A, 2019, vol.52, no. 20, 205203, $16 \mathrm{pp}$.

[26] García-Naranjo, L. C., Hamiltonisation, Measure Preservation and First Integrals of the MultiDimensional Rubber Routh Sphere, Theor. Appl. Mech., 2019, vol.46, no. 1, pp. 65-88.

[27] Jovanović, B., Note on a Ball Rolling over a Sphere: Integrable Chaplygin System with an Invariant Measure without Chaplygin Hamiltonization, Theor. Appl. Mech., 2019, vol.46, no. 1, pp.97-108.

[28] Jovanović, B., Hamiltonization and Integrability of the Chaplygin Sphere in $\mathbb{R}^{n}$, J. Nonlinear. Sci., 2010, vol. 20, no. 5, pp. 569-593.

[29] Jovanović, B., The Jacobi-Rosochatius Problem on an Ellipsoid: The Lax Representations and Billiards, Arch. Ration. Mech. Anal., 2013, vol.210, no.1, pp. 101-131.

[30] Jovanović, B., Invariant Measures of Modified LR and L+R Systems, Regul. Chaotic Dyn., 2015, vol. 20, no. 5, pp. 542-552.

[31] Jovanović, B., Noether Symmetries and Integrability in Hamiltonian Time-Dependent Mechanics, Theor. Appl. Mech., 2016, vol.43, no. 2, pp. 255-273.

[32] Jovanović, B., Symmetries of Line Bundles and Noether Theorem for Time-Dependent Nonholonomic Systems, J. Geom. Mech., 2018, vol. 10, no. 2, pp. 173-187.

[33] Jovanović, B., Rolling Balls over Spheres in $\mathbb{R}^{n}$, Nonlinearity, 2018, vol.31, no. 9, pp. 4006-4031.

[34] Koiller, J., Reduction of Some Classical Nonholonomic Systems with Symmetry, Arch. Rational Mech. Anal., 1992, vol. 118, no. 2, pp.113-148.

[35] Moser, J., Various Aspects of Integrable Hamiltonian Systems, in Dynamical Systems (C.I.M.E. Summer School, Bressanone, 1978), Progr. Math., vol.8, Boston, Mass.: Birkhäuser, 1980, pp. 233-289. 
[36] Stanchenko, S. V., Non-Holonomic Chaplygin Systems, J. Appl. Math. Mech., 1989, vol.53, no. 1, pp. 11-17; see also: Prikl. Mat. Mekh., 1989, vol. 53, no. 1, pp.16-23.

[37] Tsiganov, A. V., Integrable Euler Top and Nonholonomic Chaplygin Ball, J. Geom. Mech., 2011, vol. 3, no. 3, pp. 337-362.

[38] Tsiganov, A. V., Integrable Discretization and Deformation of the Nonholonomic Chaplygin Ball, Regul. Chaotic Dyn., 2017, vol. 22, no. 4, pp. 353-367.

[39] Veselov, A.P. and Veselova, L. E., Flows on Lie Groups with a Nonholonomic Constraint and Integrable Non-Hamiltonian Systems, Funct. Anal. Appl., 1986, vol. 20, no. 5, pp. 308-309; see also: Funktsional. Anal. i Prilozhen., 1986, vol. 20, no. 4, pp. 65-66.

[40] Veselov, A.P. and Veselova, L.E., Integrable Nonholonomic Systems on Lie Groups, Math. Notes, 1988, vol.44, no. 5, pp.810-819; see also: Mat. Zametki, 1988, vol.44, no. 5, pp. 604-619. 